\title{
Computational Static Aeroelasticity Using Nonlinear Structures and Aerodynamics Models
}

\author{
Hitoshi Arizono* \\ Japan Aerospace Exploration Agency, Mitaka, Tokyo, 181-0015, Japan \\ Carlos E. S. Cesnik ${ }^{\dagger}$ \\ The University of Michigan, Ann Arobor, MI, 48109, USA
}

\begin{abstract}
This paper describes the development of structural solver based on the corotational approach using the three-node triangular shell element and the integration of existing aeroelastic solver. The static nonlinear aeroelastic responses of a high aspect ratio wing are also presented.
\end{abstract}

\section{Introduction}

High-Altitude Long-Endurance (HALE) aircraft has high aspect ratio wings to achieve the mission requirements. HALE aircraft demands high aerodynamic and structural efficiency because of lightweight and flexibility. The wings may undergo large deformations and appear the geometrically nonlinear behavior. Nonlinear structural as well as aerodynamic modeling are necessary for the aeroelastic analysis and design of HALE aircraft.

The aeroelasticity of HALE aircraft has been widely studied and several nonlinear aeroelastic solvers have been developed. Van Schoor and von Flotow ${ }^{1}$ studied very flexible aircraft using linear finite element analysis and $2 \mathrm{D}$ unsteady strip theory aerodynamics. Their results indicate that unsteady aerodynamics and flexibility of the aircraft should be considered so as to correctly model the dynamic system. Patil,Hodges, and Cesnik $^{2}$ investigated the aeroelasticity and flight dynamics of HALE aircraft. They modeled a very flexible wing as an exact intrinsic beam model with Peter's finite-state aerodynamics. The results indicate the overall flight dynamic characteristics of the aircraft change due to wing flexibility. Drela ${ }^{3}$ developed an integrated design and analysis package which incorporated a nonlinear beam model with lifting-line aerodynamics. Cesnik and his co-workers have developed Nonlinear Aeroelastic Simulation Toolbox (UM/NAST) ${ }^{4}$ which uses a strain-based structural formulation and Peter's finite-state aerodynamics. Several aeroealstic issues in HALE aircraft have been addressed: nonlinear aeroelastic modeling, ${ }^{4,5}$ integral wing actuation for generating maneuver loads, ${ }^{6}$ flutter boundary enhancement, ${ }^{7}$ gust load alleviation, ${ }^{8}$ and overall nonlinear vehicle optimization of unconventional configuration. ${ }^{6}$ Garcia et al. ${ }^{9,10}$ studied the the effects of transonic aerodynamics of a slender wing by coupling a Navier-Stokes solver with a nonlinear beam model. Smith et al. ${ }^{11}$ coupled an Euler solver with a geometrically exact beam model to investigate the effect of nonlinear aerodynamics and structures compared to various linear solutions. They concluded that linear aerodynamics theories result in larger steady state displacements and conservative flutter predictions. Palacios and Cesnik $^{12}$ coupled a nonlinear, quasi-3D structural solver with ENS3DAE to investigate static aeroelasticity of HALE wings in compressible flow. Hallissy and Cesnik ${ }^{13}$ studied static and dynamic aeroelasticity of HALE wings using CFD code coupled quasi-3D, slender structural model.

These solvers use the nonlinear beam models for structural descretization. This paper describes the development of structural solver based on the corotational approach using the three-node triangular shell element and the integration of existing aeroelastic solver. The static nonlinear aeroelastic responses of a high aspect ratio wing are also presented.

\footnotetext{
*Researcher, Aerospace Research and Development Directorate, and AIAA Senior Member.

${ }^{\dagger}$ Professor, Department of Aerospace Engineering, and AIAA Fellow.
} 


\section{Structural Solver}

The three-node triangular shell element which is combined with the optimal membrane (OPT) element ${ }^{14}$ and discrete Kirchhoff (DKT) plate bending element ${ }^{15}$ is used for the finite element discretization of model. The nodal displacement vector and the stiffness matrix of OPT element are as follows:

$$
\begin{gathered}
\left\{d_{m}\right\}=\left\{\begin{array}{lllllllll}
u_{1} & v_{1} & \theta_{z 1} & u_{2} & v_{2} & \theta_{z 2} & u_{3} & v_{3} & \theta_{z 3}
\end{array}\right\}^{T} \\
{\left[k_{m}\right]=\frac{1}{V}[L][E][L]^{T}+\int_{\Omega}\left[B_{m}\right]^{T}[E]\left[B_{m}\right] d A}
\end{gathered}
$$

where $[E]$ is the elasticity matrix, $\left[B_{m}\right]$ is the strain-displacement matrix, $[L]$ is the constant matrix, and $V$ is the volume of element. The nodal displacement vector and the stiffness matrix of DKT element are as follows:

$$
\begin{aligned}
& \left\{d_{b}\right\}=\left\{\begin{array}{lllllllll}
w_{1} & \theta_{x 1} & \theta_{y 1} & w_{2} & \theta_{x 2} & \theta_{y 2} & w_{3} & \theta_{x 3} & \theta_{y 3}
\end{array}\right\}^{T} \\
& {\left[k_{b}\right]=\int_{\Omega}\left[B_{b}\right]^{T}\left[D^{e}\right]\left[B_{b}\right] d A=2 A \int_{0}^{1} \int_{0}^{1-\zeta_{3}}\left[B_{b}\right]^{T}\left[D^{e}\right]\left[B_{b}\right] d \zeta_{2} d \zeta_{3}}
\end{aligned}
$$

where $\left[D^{e}\right]$ is the flexural rigidity of the plate, $\left[B_{b}\right]$ is the strain-displacement vector, $A$ is the area of the element. The stiffness matrices of OPT element and DKT element are combined to form the shell stiffness matrix of the element. The nodal displacement vector and the stiffness matrix of shell element are as follows:

$$
\begin{gathered}
\left\{\begin{array}{c}
\left\{d_{m}\right\} \\
\left\{d_{b}\right\}
\end{array}\right\}=\left\{\begin{array}{lllllllll}
\left\{u_{1}\right. & v_{1} & \theta_{z 1} & u_{2} & v_{2} & \theta_{z 2} & u_{3} & v_{3} & \left.\theta_{z 3}\right\}^{T} \\
\left\{w_{1}\right. & \theta_{x 1} & \theta_{y 1} & w_{2} & \theta_{x 2} & \theta_{y 2} & w_{3} & \theta_{x 3} & \left.\theta_{y 3}\right\}^{T}
\end{array}\right\} \\
{[k]\{d\}=\left[\begin{array}{cc}
{\left[k_{m}\right]_{9 \times 9}} & 0 \\
0 & {\left[k_{b}\right]_{9 \times 9}}
\end{array}\right]\left\{\begin{array}{c}
\left\{d_{m}\right\} \\
\left\{d_{b}\right\}
\end{array}\right\}}
\end{gathered}
$$

In order to have the tangent stiffness, the following stress stiffness matrix is added to the shell stiffness matrix.

$$
\left[k_{\sigma}\right]=\int[G]^{T}\left[\begin{array}{ccc}
{[\bar{N}]} & 0 & 0 \\
0 & {[\bar{N}]} & 0 \\
0 & 0 & {[\bar{N}]}
\end{array}\right][G] d x d y
$$

This integral can be evaluated exactly using seven Gauss points in the area coordinate system.

In corotational approach, ${ }^{16}$ the total motion of an element is decomposed into a rigid body motion and a pure deformation. Then the contribution of the rigid body motion to the total deformation of the element is removed before performing the element computations. This will enable to upgrade the structural elements to treat problems with large rotation but small strain. Figure 1 shows the initial (undeformed) and current configurations of a general triangular shell element, moving in the global coordinate system $g$.

The displacement vector of node $i$ with position vector $X_{i}^{G}$ in the global coordinate is denoted by $u_{i}^{g}$. $E_{0}$ is the local coordinate system in the initial configuration with the origin at node 1 and axis $E_{01}$ along the side $1-2$ and $E_{03}$ perpendicular to the element plane. In the current configuration, the position vector of node $i$ is given by $x_{i}^{g}=X_{i}^{g}+u_{i}^{g}$. Using $x_{i}^{g}$, local coordinate system $E$ in the current configuration is established.

Rotations of each node in the global coordinate system are expressed by a triad which is rigidly tied to the node and rotates with the node from its initial state $S_{0}$ to its current state $S$. Updating triad $S$ for incremental rotations after each iteration is performed by updating its transformation matrix $T_{S}$. Assuming $\tilde{\theta}_{X}, \tilde{\theta}_{Y}$ and $\tilde{\theta}_{Z}$ as the incremental rotations of triad $S$ resulted from the last iteration computed in global coordinate system, $T_{S}$ may be updated by the following expression:

$$
\left(T_{S}\right)_{\text {new }}=\tilde{T} \cdot\left(T_{S}\right)_{\text {old }}
$$

where

$$
\tilde{T}=I+\frac{\tilde{\Omega}+0.5 \tilde{\Omega}^{2}}{1+0.25|\omega|^{2}}, \quad|\omega|=\sqrt{\tilde{\theta}_{X}^{2}+\tilde{\theta}_{Y}^{2}+\tilde{\theta}_{Z}^{2}}, \quad \tilde{\Omega}=\left[\begin{array}{ccc}
0 & -\tilde{\theta}_{Z} & \tilde{\theta}_{Y} \\
\tilde{\theta}_{Z} & 0 & -\tilde{\theta}_{X} \\
-\tilde{\theta}_{Y} & \tilde{\theta}_{X} & 0
\end{array}\right]
$$




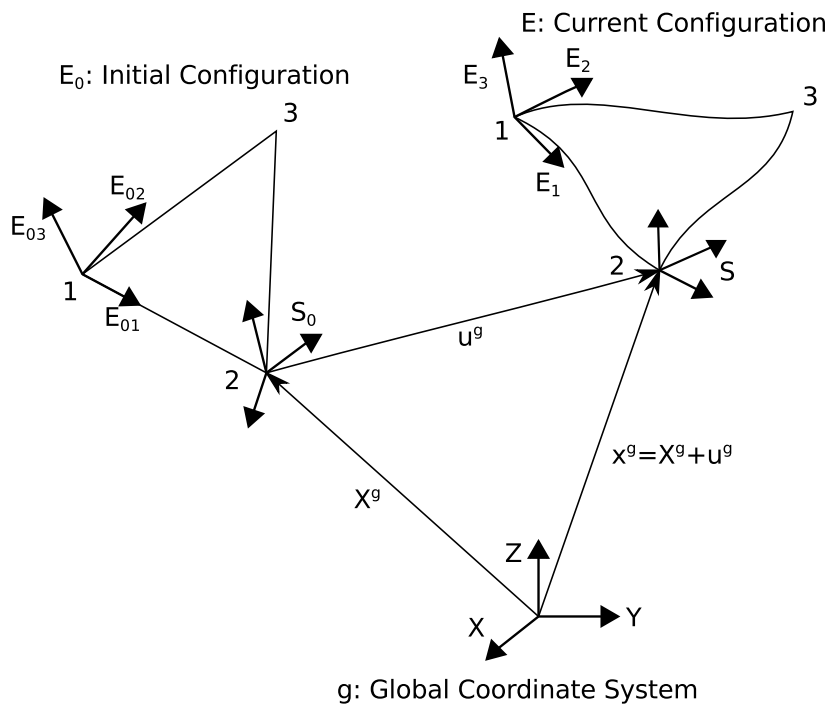

Figure 1. Corotation coordinate.

Pure nodal rotations in local coordinate system $E$ are equal to components of an antisymmetric matrix $\Omega$ as:

$$
\Omega=\left[\begin{array}{ccc}
0 & -\theta^{E_{3}} & \theta^{E_{2}} \\
\theta^{E_{3}} & 0 & -\theta^{E_{1}} \\
-\theta^{E_{2}} & \theta^{E_{1}} & 0
\end{array}\right]
$$

where $\Omega$ is found by following expression using orthogonal matrix $T=T_{E}^{T} T_{S} T_{E 0}$ :

$$
\Omega=2(T-I)(T+I)^{-1}
$$

Pure nodal displacements at node $i$ in local coordinate system $E$ are as follows:

$$
\bar{u}_{i}^{E}=\left\{\begin{array}{l}
\bar{u}_{i}^{E_{1}} \\
\bar{u}_{i}^{E_{2}} \\
\bar{u}_{i}^{E_{3}}
\end{array}\right\}=T_{E}^{T}\left(u_{i}^{g}+X_{i}^{g}-u_{1}^{g}-X_{1}^{g}\right)-X_{i}^{E_{0}}, \quad i=1,2,3
$$

where $X_{i}^{E_{0}}$ is the initial coordinates of node $i$ in $E_{0}$. Finally, the pure deformations at node $i$ computed in local coordinate system $E$ can be expressed as:

$$
\left\{d_{i}\right\}_{\text {pure }}=\left\{\begin{array}{llllll}
\bar{u}_{i}^{E_{1}} & \bar{u}_{i}^{E_{2}} & \bar{u}_{i}^{E_{3}} & \theta_{i}^{E_{1}} & \theta_{i}^{E_{2}} & \theta_{i}^{E_{3}}
\end{array}\right\}^{T}, \quad i=1,2,3
$$

These pure deformations may not be really pure and a projector matrix $P^{17}$ can be introduced to bring the non-equilibrated internal force vector into equilibrium. The local element stiffness matrix and the internal force vector are computed as follows:

$$
\{\bar{d}\}=[P]\{d\}, \quad\{\bar{r}\}=[P]^{T}\{r\}=[P]^{T}[k]\{\bar{d}\}, \quad[\bar{k}]=[P]^{T}[k][P]
$$

Transforming $[\bar{k}]$ and $\{\bar{r}\}$ to the global coordinate system, the procedure is repeated for all elements to assemble current structural stiffness $[\bar{K}]$ and internal force $\left\{R^{i n t}\right\}$. Load imbalance $\{\Delta R\}$ is found by subtracting $\left\{\bar{R}^{\text {int }}\right\}$ from the vector of applied load $\left\{R^{e x t}\right\}$. The structure equations are as follows:

$$
[\bar{K}]\{\Delta D\}=\{\Delta R\}
$$

For the static aeroelastic simulation, this equation can be solved using Newton-Raphson method for displacement increment $\{\Delta \bar{D}\}$. 
The structural dynamics governing equations of motion can be written as:

$$
[M]\{\ddot{D}\}+[C]\{\dot{D}\}+\left\{R^{\text {int }}\right\}=\left\{R^{\text {ext }}\right\}
$$

The numerical integration of the governing equations is performed using the Newmark or the generalized- $\alpha$ methods. ${ }^{18}$ The Newmark relations to update the displacements and velocities are as follows:

$$
\begin{gathered}
\{D\}_{n+1}=\{D\}_{n}+\Delta t\{\dot{D}\}_{n}+\Delta t^{2}\left[\left(\frac{1}{2}-\beta\right)\{\ddot{D}\}_{n}+\beta\{\dot{D}\}_{n+1}\right] \\
\{\dot{D}\}_{n+1}=\{\dot{D}\}_{n}+\Delta t\left[(1-\gamma)\{\ddot{D}\}_{n}+\gamma\{\ddot{D}\}_{n+1}\right]
\end{gathered}
$$

The balanced equations for generalized- $\alpha$ method are given by:

$$
[M]\{\ddot{D}\}_{n+1-\alpha_{m}}+[C]\{\dot{D}\}_{n+1-\alpha_{f}}+\left\{R^{i n t}\right\}_{n+1-\alpha_{f}}=\left\{R^{e x t}\right\}_{n+1-\alpha_{f}}
$$

Using the Newmark update relations, the balanced equation is rearranged as follows:

$$
\begin{gathered}
{\left[K^{\mathrm{eff}}\right]\{\Delta D\}=\left\{R^{\mathrm{eff}}\right\}} \\
\left\{K^{\mathrm{eff}}\right]=\frac{1-\alpha_{m}}{\beta \Delta t^{2}}[M]+\frac{\left(1-\alpha_{f}\right) \gamma}{\beta \Delta t}[C]+\left(1-\alpha_{f}\right)\left[K_{t}\right] \\
\left\{R^{\mathrm{eff}}\right\}=[M] \frac{1-\alpha_{m}}{\beta \Delta t^{2}} \Delta t_{s}\{\dot{d}\}_{n}+[M] \frac{1-\alpha_{m}}{\beta \Delta t^{2}} \Delta t^{2}\left(\frac{1}{2}-\beta\right)\{\ddot{d}\}_{n}-[M] \alpha_{m}\{\ddot{d}\}_{n} \\
-[C]\left(1-\alpha_{f}\right)\{\dot{d}\}_{n}-[C]\left(1-\alpha_{f}\right) \Delta t(1-\gamma)\{\ddot{d}\}_{n}+[C]\left(1-\alpha_{f}\right) \frac{\gamma}{\beta}\{\dot{d}\}_{n} \\
+[C]\left(1-\alpha_{f}\right) \frac{\gamma \Delta t}{\beta}\left(\frac{1}{2}-\beta\right)\{\ddot{d}\}_{n}-\left(1-\alpha_{f}\right)\left\{R^{\mathrm{int}}\right\}_{n}-\alpha_{f}\left\{R^{\mathrm{int}}\right\}_{n} \\
+\left(1-\alpha_{f}\right)\left\{R^{\mathrm{ext}}\right\}_{n+1}+\alpha_{f}\left\{R^{\mathrm{ext}}\right\}_{n}
\end{gathered}
$$

\section{Aerodynamic Solver}

The computation of the aerodynamic forces is used existing aeroelastic solver which has been developed at JAXA. This solver is based on cell-centered, finite volume scheme on multiblock structured grid. The governing equations for the flow field can be written as:

$$
\frac{\partial Q}{\partial t}+\frac{\partial E}{\partial x}+\frac{\partial F}{\partial y}+\frac{\partial G}{\partial z}=\frac{1}{\operatorname{Re}}\left\{\frac{\partial E_{v}}{\partial x}+\frac{\partial F_{v}}{\partial y}+\frac{\partial G_{v}}{\partial z}\right\}
$$

where

$$
\begin{aligned}
& Q=\left(\begin{array}{c}
\rho \\
\rho u \\
\rho v \\
\rho w \\
e
\end{array}\right), \quad E=\left(\begin{array}{c}
\rho u \\
\rho u^{2}+p \\
\rho u v \\
\rho u w \\
(e+p) u
\end{array}\right), \quad F=\left(\begin{array}{c}
\rho v \\
\rho u v \\
\rho v^{2}+p \\
\rho v w \\
(e+p) v
\end{array}\right), \quad G=\left(\begin{array}{c}
\rho w \\
\rho u w \\
\rho v w \\
\rho w^{2}+p \\
(e+p) w
\end{array}\right) \\
& E_{v}=\left(\begin{array}{c}
0 \\
\tau_{x x} \\
\tau_{x y} \\
\tau_{x z} \\
\beta_{x}
\end{array}\right), \quad F_{v}=\left(\begin{array}{c}
0 \\
\tau_{y x} \\
\tau_{y y} \\
\tau_{y z} \\
\beta_{y}
\end{array}\right), \quad G_{v}=\left(\begin{array}{c}
0 \\
\tau_{z x} \\
\tau_{z y} \\
\tau_{z z} \\
\beta_{z}
\end{array}\right)
\end{aligned}
$$




\section{Numerical Results}

\section{A. Validation of structural solver}

A cantilever plate made of an isotropic material subject to an end moment is considered (Figure 2). The plate size is $0.6 \mathrm{~m} \times 0.3 \mathrm{~m} \times 0.01 \mathrm{~m}$. The material properties are Young's modulus $E=196.2 \mathrm{GPa}$, shear modulus $G=75.46 \mathrm{GPa}$, and Poisson's ratio $\nu=0.3$. Figure 3 shows the transverse tip deflection against the applied moment, and current solution is good agreement with the exact solution. ${ }^{16}$



Figure 2. Cantilever plate subjected to end moment.

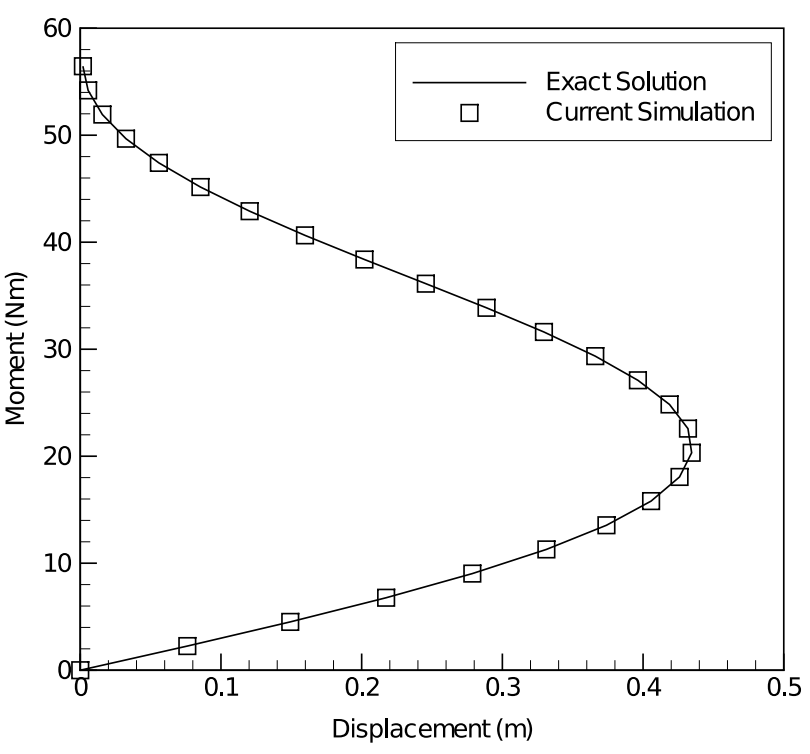

Figure 3. Tip displacement as a function of the applied moment.

\section{B. Static aeroelastic simulation}

Using the developed solver, the static and aeroelastic simulations of the high aspect ratio wing (Figure 6) were conducted. The chord length is $1 \mathrm{~m}$ and the span length is $16 \mathrm{~m}$. The airfoil section is NACA0012. The structural properties are Young's modulus $E=200 \mathrm{GPa}$, shear modulus $G=100 \mathrm{GPa}$, and Poisson's ratio $\nu=0.3$. The structural mesh and the aerodynamic mesh are shown in Figure 4 and 5.

Figure 6 shows the static aeroelastic deformation and the pressure coefficient contour for Mach number $M=0.30$ at angle of attack $\alpha=2.0 \mathrm{deg}$.

The tip displacements are compared with results of reference ${ }^{13}$ in Figure 7 . Both the bending displacement and twist angle of current results are larger than other results. The in-plane bending deformation appears in our results becase the isotropic material is used in our model. On the other hands, the rigidity of chord-wise is larger than that of span-wise in reference. ${ }^{13}$ As a result, it is assumed that the larger displacements of bending and twist appears because of the small torsional rigidity.

\section{Concluding Remarks}

The structural solver based on the three-node triangular element with the corotational approach was developed. This structural solver was combined the existing aeroelastic solver, and the static aeroelastic simulations of a high aspect ratio wing were conducted. These solver is possible to use in high-fidelity nonlinear aeroelastic analysis of complex configuration in the time domain. Further studies will focus on dynamic aeroelastic simulations. 


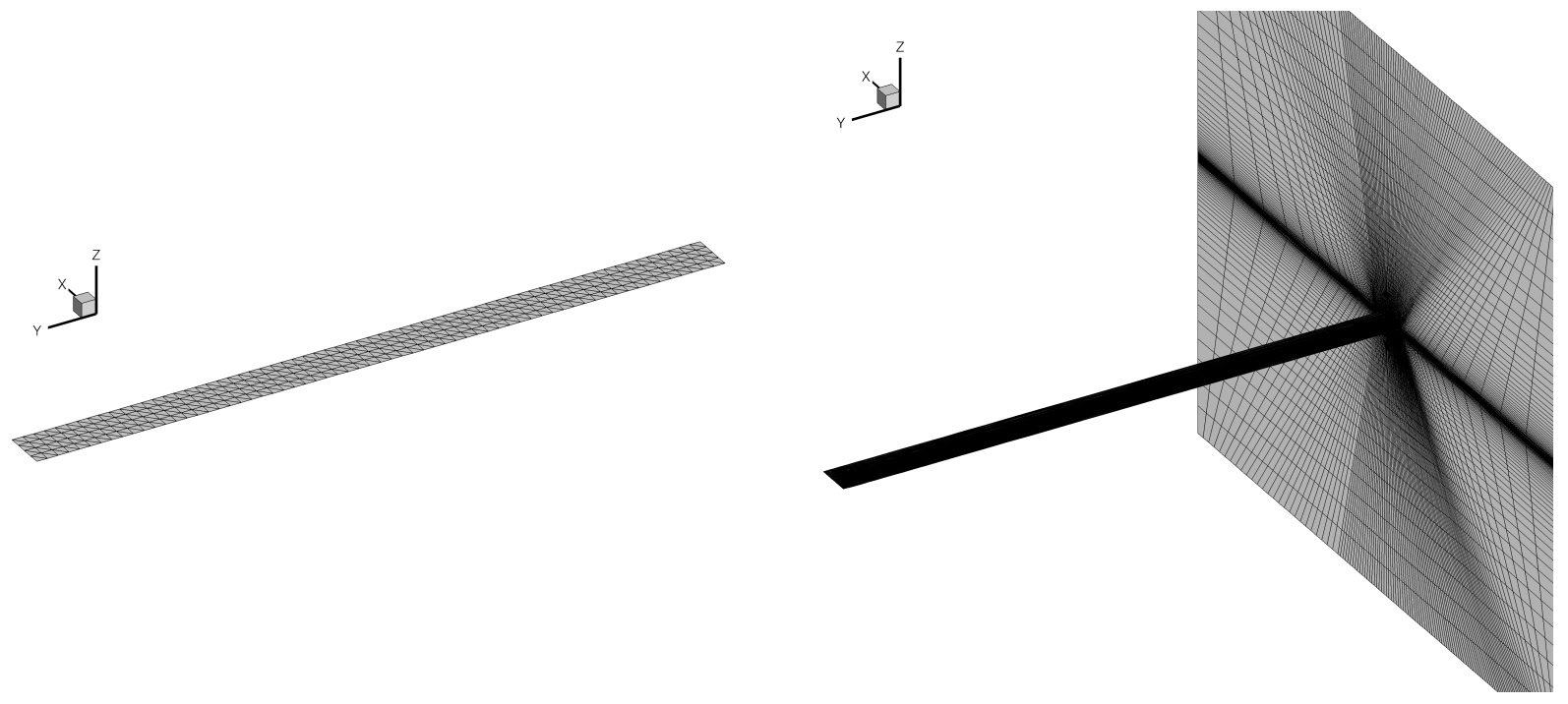

Figure 4. FEM mesh.

Figure 5. CFD mesh.



Figure 6. Static aeroelastic deformation and pressure coefficient contour.

\section{References}

${ }^{1}$ M. C. van Schoor and A. H. von Flotow, "Aeroelastic Characteristics of a Highly Flexible Aircraft," AIAA Journal, Vol. 27, No. 10, pp. 901908, October 1990.

${ }^{2}$ M. J. Patil, D. H. Hodges, and C. E. S. Cesnik, "Nonlinear Aeroelastic Analysis of Aircraft with High-Aspect-Ratio Wings," Proceedings of 39th AIAA/ASME/ASCE/AHS/ASC Structures, Structural Dynamics and Materials Conference, AIAA Paper 98-1955, Long Beach, CA, April 1998.

${ }^{3}$ M. Drela, "Integrated Simulation Model for Preliminary Aerodynamic, Structural, and Control-Law Design of Aircraft," Proceedings of the 40th AIAA/ASME/ASCE/AHS/ASC Structures, Structural Dynamics, and Materials Conference and Exhibit, AIAA Paper 99-1394, St. Louis, MO, April 1999.

${ }^{4}$ C. E. S. Cesnik and W. Su, "Nonlinear Aeroelastic Modeling and Analysis of Fully Flexible Aircraft," Proceedings of the 46th AIAA/ASME/ASCE/AHS/ASC Structures, Structural Dynamics, and Materials Conference, AIAA Paper 2005-2169, Austin, TX, April 2005.

${ }^{5}$ C. M. Shearer and C. E. S. Cesnik, "Nonlinear Flight Dynamics of Very Flexible Aircraft," Journal of Aircraft, vol. 44, no. 5, pp. 15281545, September-October 2007.

${ }^{6}$ C. E. S. Cesnik and E. L. Brown, "Active Warping Control of a Jointed-Wing Airplane Configuration," Proceedings of the 44th AIAA/ASME/ASCE/AHS/ASC Structures, Structural Dynamics, and Materials Conference, AIAA Paper 2003-1715, Norfolk, VA, April 2003.

${ }^{7}$ C. E. S. Cesnik and M. Ortega-Morales, "Active Aeroelastic Tailoring of Slender Flexible Wings," Proceedings of the International Forum on Aeroelasticity and Structural Dynamics, Madrid, Spain, 2001.

${ }^{8}$ W. Su and C. E. S. Cesnik, "Dynamic Response of Highly Flexible Flying Wings," Proceedings of the 47th 

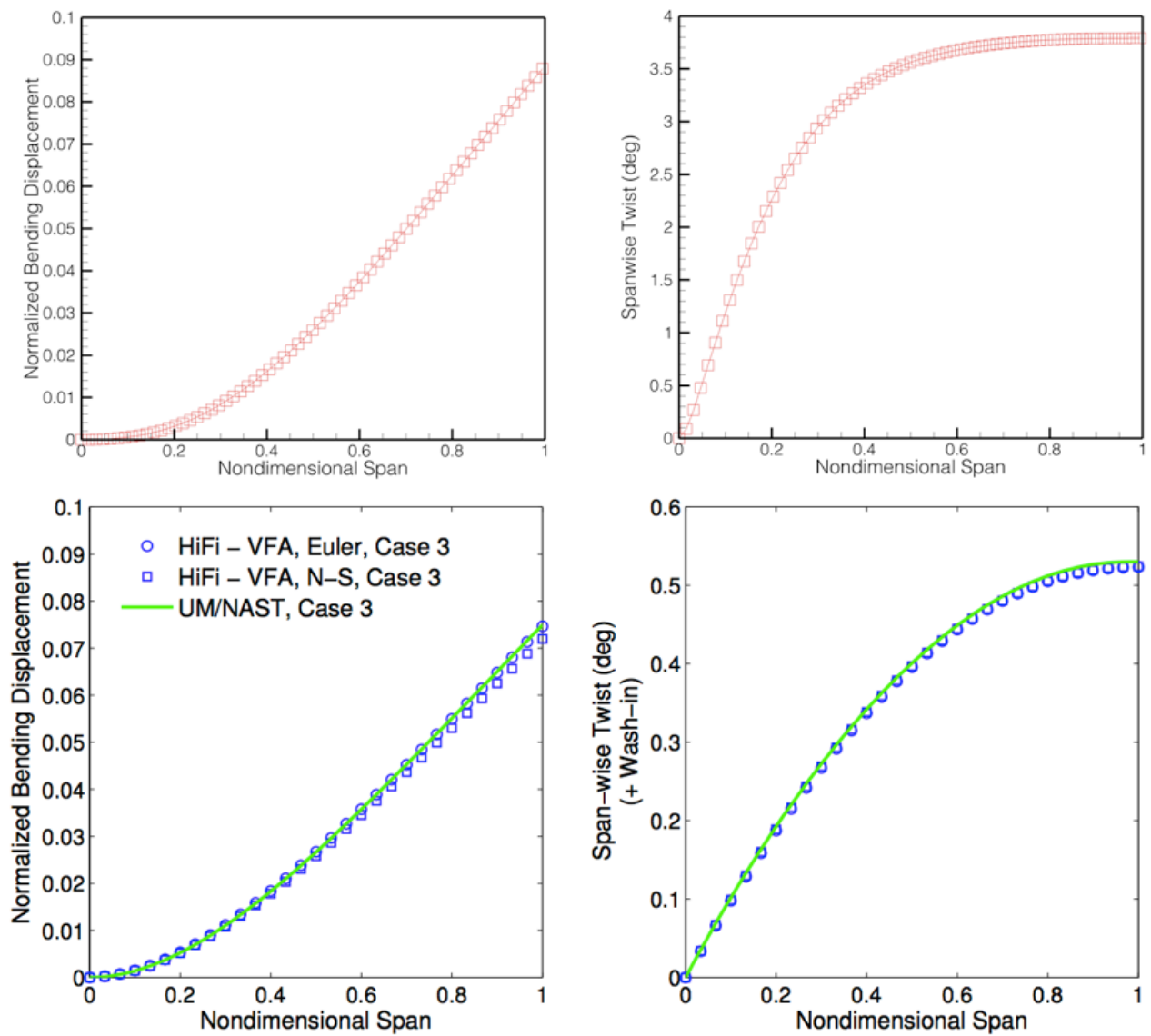

Figure 7. Static aeroelastic displacements. (Top: current simulation results / Bottom: Ref. ${ }^{13}$ )

AIAA/ASME/ASCE/AHS/ASC Structures, Structural Dynamics, and Materials Conference, AIAA Paper 2006-1636, Newport, RI, May 2006.

${ }^{9}$ J. A. Garcia and G. P. Guruswamy, "Aeroelastic Analysis of Transonic Wings Using Navier-Stokes Equations and a Nonlinear Beam Finite Element Model," Proceedings of the 40th AIAA/ASME/ASCE/AHS/ASC Structures, Structural Dynamics, and Materials Conference and Exhibit, AIAA Paper 99-1215, St. Louis, MO, April 1999.

${ }^{10}$ J. A. Garcia, "Numerical Investigation of Nonlinear Aeroelastic Effects on Flexible High-Aspect-Ratio Wings," Journal of Aircraft, Vol. 42, No. 4, pp. 10251036, July-August 2005.

${ }^{11}$ M. J. Smith, M. J. Patil, and D. H. Hodges, "CFD-Based Analysis of Nonlinear Aeroelastic Behavior of High-Aspect Ratio Wings," Proceedings of the 42nd AIAA/ASME/ASCE/AHS/ASC Structures, Structural Dynamics, and Materials Conference and Exhibit, AIAA Paper 2001-1582, Seattle, WA, April 2001.

${ }^{12}$ R. Palacios and C. E. S. Cesnik, "Static Nonlinear Aeroelasticity of Flexible Slender Wings in Compressible Flow," Proceedings of the 46th AIAA/ASME/ASCE/AHS/ASC Structures, Structural Dynamics, and Materials Conference, AIAA Paper 2005-1945, Austin, TX, April 2005.

${ }^{13}$ B. P. Hallissy and C. E. S. Cesnik, "High-fidelity Aeroelastic Analysis of Very Flexible Aircraft," AIAA Paper 2011-1914.

${ }^{14}$ C. A. Felippa, "A study of optimal membrane triangles with drilling freedoms," Computer Methods in Applied Mechanics and Engineering, Vol. 192, No. 16, pp. 21252168, April 2003.

${ }^{15}$ J.-L. Batoz, K.-J. Bathe, and L.-W. Ho, "A Study of Three-Node Triangular Plate Bending Elements," International Journal for Numerical Methods in Engineering, Vol. 15, pp. 17711812, 1980.

${ }^{16}$ P. Khosravi, R. Ganesan, and R. Sedaghati, "Corotational non-linear analysis of thin plates and shells using a new shell element," International Journal for Numerical Methods in Engineering, Vol. 69, No. 4, pp. 859885, 2007.

${ }^{17}$ C. C. Rankin and B. Nour-Omid, "The Use of Projectors to Improve Finite Element Performance," Computers \& Structures, Vol. 30, No. 1, pp. 257267, 1988.

${ }^{18}$ J. Chung and G. M. Hulbert, "A Time Integration Algorithm for Structural Dynamics with Improved Numerical Dissipation: The Generalized- $\alpha$ Method," Journal of Applied Mechanics, Vol. 60, pp. 371375, Jun. 1993. 